\title{
Study on the effect of different muscle fatigue recovery methods on muscle stiffness recovery
}

\author{
Fan Zhang 1, 2, a, Aimin Zhou 1,b \\ 1Department of Police Skills and Tactics, Nanjing Forest Police College, Nanjing 210023, China; \\ ${ }^{2}$ Sports Science postdoctoral programme, Nanjing Normal University, Nanjing 210023, China. \\ azhangfan@nfpc.edu.cn, bzhouaimin@nfpc.edu.cn
}

Keywords: muscle stiffness; muscle fatigue; muscle recovery; concentric contraction; eccentric contraction.

\begin{abstract}
The purpose of this study was to determine the effects of several passive recovery (PR), active recovery (AR), vibration recovery (VR) and cryotherapy recovery $(\mathrm{CR})$ on the recovery of muscle stiffness (MS). 20 healthy male college students were enrolled in the study. MS of the biceps brachii of the non-handedness was measured. The differences and recovery effects of MS at different detection time points were tested by one-way repeated measures ANOVA. The results indicated that the muscle stiffness in different time points did not change significantly, the reason for the fatigue movement in a short period of time on muscle stiffness caused no obvious difference, or is this study using the sensitivity of muscle tension is not sensitive.
\end{abstract}

\section{Introduction}

When the human body moves, the muscle activity is the core, and other organs in the body, system function changes, all in order to ensure the realization of muscle activity [1], but overexercise or game with furious competition may cause physical fatigue, and especially, muscle fatigue sourced from continuous muscle contraction will lower late exercise performance and bring muscle injury response. The soreness of the muscles is associated with the contraction of the muscles and this constant muscle tension causes the stiffness of the muscles to increase [2]. This phenomenon is likely to block blood circulation in muscle tissue. Many studies have shown that muscle contraction during ischemia can cause pain. Therefore, muscle soreness is a manifestation of muscle fatigue, muscle fatigue leads to muscle stiffness, and it will have adverse effects on individual activities [3]. therefore, if effective recovery method is used within limited time, it will be quite beneficial to re-performance improvement and exercise injury reduction.

\section{Research object and method}

\subsection{Object of study.}

20 male undergraduates having healthy body are subjects (they are free from cardiovascular disease, high blood pressure and relevant viscera disease, and they can receive the maximum exercise test), with average age $(20.4 \pm 2.3 \mathrm{yrs}$.), average height $(173.5 \pm 3.4 \mathrm{~cm})$ and average weight $(68.7 \pm 3.9 \mathrm{~kg})$.

\subsection{Experimental design.}

Experimental design of repetitive measurement is adopted, and every subject accepts four different fatigue recovery ways in anti-balance sequence method: PR, AR, VR, CR. Interval of experimental procedure in every step shall be over 7 days to avoid that delayed onset muscle soreness disturbs experimental result.

1) In phase of familiarization with actions, elbow joint shall bend to contract 10 times centripetally and eccentrically repeatedly in $45 \%$ of angular speed;

2) In phase of measurement before fatigue, BBM Muscle Stiffness (MS) shall be measured in sequence to avoid that mechanical property is affected by muscle manifestation test. 
3) In phase of muscle fatigue, isokinetic exercise system of Biodex System Pro-4 is adopted, and maximum contraction exercise shall be conducted centripetally and eccentrically repeatedly in $45 \%$ of angular speed on bent of non-habitual elbow joint, and activity scope is $30 \sim 120^{\circ}$ (it is $0^{\circ}$ when elbow completely stretches straightly), and muscles can be judged in fatigue until finally there are continual three times where muscle force manifestation reduces to $50 \% \mathrm{MVC}$, and then exercise of constant speed can be stopped.

4) In phase of measurement after fatigue, data of MS shall be immediately measured.

5) In phase of intervening of recovery way, one of its recovery ways can be conducted in random way: PR group shall rest for 10 minutes in still sitting gesture; concentric contraction exercise shall be conducted for AR group in constant speed on isokinetic exercise system of Biodex System Pro-4. It shall start in strength of $25 \% \mathrm{MVC}$, and the speed shall decrease by $5 \%$ MVC every two minutes, and there shall be 10 minutes totally. For VR group, vibration training platform Power Plate Pro5 AIR ${ }^{\mathrm{TM}}$ shall be used to partially vibrate BBM, and vibration frequency shall be $30 \mathrm{~Hz}$, amplitude being $2 \mathrm{~mm}$ for 10 minutes. Ice compression shall be conducted on fatigue parts with LP ice pack (LP894; M: $22.9 \mathrm{~cm}$; $65 \%$ ice block filling) non-pressure device for 10 minutes for CR group.

6) In phase of measurement after recovery, MS of BBM shall be measured immediately.

7) Then experiment of another recovery way shall be conducted until totally recovery with interval over 7 days.

\subsection{Test data and collection.}

1) Muscle stiffness. MS value of BBM at different time points can be measured via myotonometer (R)-muscle state measuring system. The experimenter grasps the handle and advances BBM vertically via designed external pressure $(2 \mathrm{~kg})$, and plexiglass ring shall be fixed on skin surface. Pressure change can be monitored via displacement caused by change of probe and plexiglass ring for converter. Data can be recorded after mean value is chosen for five experiments, and MS can be judged via muscle deformation degree.

2) Recovery effect. Values of MS at different measurement time points (before fatigue, after fatigue and after recovery) shall be calculated, and then [(after recovery/before fatigue) $\times 100 \%$-(after fatigue/before fatigue) $\times 100 \%$ ] shall be deemed as recovery effect of MS.

\subsection{Statistical analysis.}

Statistical handling shall be conducted via SPSS 19.0, and the result shall be represented with mean value \pm standard deviation $(\bar{X} \pm \mathrm{SD})$. Difference of $\mathrm{MS}$ in all groups (PR, AR, VR, CR) at different time points (before fatigue, after fatigue, after recovery) shall be compared respectively via repeated measures of a single factor. Difference among groups of MS can also be compared, and posterior comparison can be conducted via way of Bonferroni if standard reaching is significant. Statistical significance level is $P<0.05$, and non-significance level is $P<0.01$.

\section{Change of Muscle stiffness after intervening of different recovery mode}

\subsection{Difference of different time points.}

It is indicated in Table 4 that only MS of PR group and AR group reach significant relationship ( $P$ $<0.05)$ at different time points, and MS of PR after recovery is significantly more than that before fatigue $(P<0.05)$ after posterior comparison, but there is no significant difference between posterior fatigue and posterior recovery. MS after recovery is significantly more than that after fatigue $(P<0.05)$ for AR(Table 1). 
Table 1. MS of different recovery methods at different time points

\begin{tabular}{cccc}
\hline MS $(\mathrm{mm} / 2 \mathrm{~kg})$ & before fatigue & after fatigue & after recovery \\
\hline PR & $11.11 \pm 1.52$ & $11.49 \pm 1.65$ & $11.72 \pm 1.42^{\mathrm{b}}$ \\
AR & $11.62 \pm 1.94$ & $11.14 \pm 1.58$ & $11.59 \pm 1.40^{\mathrm{c}}$ \\
VR & $11.41 \pm 1.85$ & $11.69 \pm 1.93$ & $11.59 \pm 1.36$ \\
CR & $11.75 \pm 1.53$ & $11.67 \pm 2.06$ & $11.56 \pm 1.52$ \\
\hline
\end{tabular}

Note: $b$ represents significant difference before fatigue and after recovery; c represents significant difference after fatigue and recovery. $P<0.05$.

\subsection{Difference of recovery effect.}

It is indicated in Fig. 1 that recovery effects among groups of $\operatorname{PR}(2.27 \pm 8.67 \%), \operatorname{AR}(3.86 \pm 5.39 \%)$, $\operatorname{VR}(0.11 \pm 14.47 \%)$ and $\operatorname{CR}(-0.67 \pm 8.96 \%)$ all do not reach significant difference $(P>0.05)$.

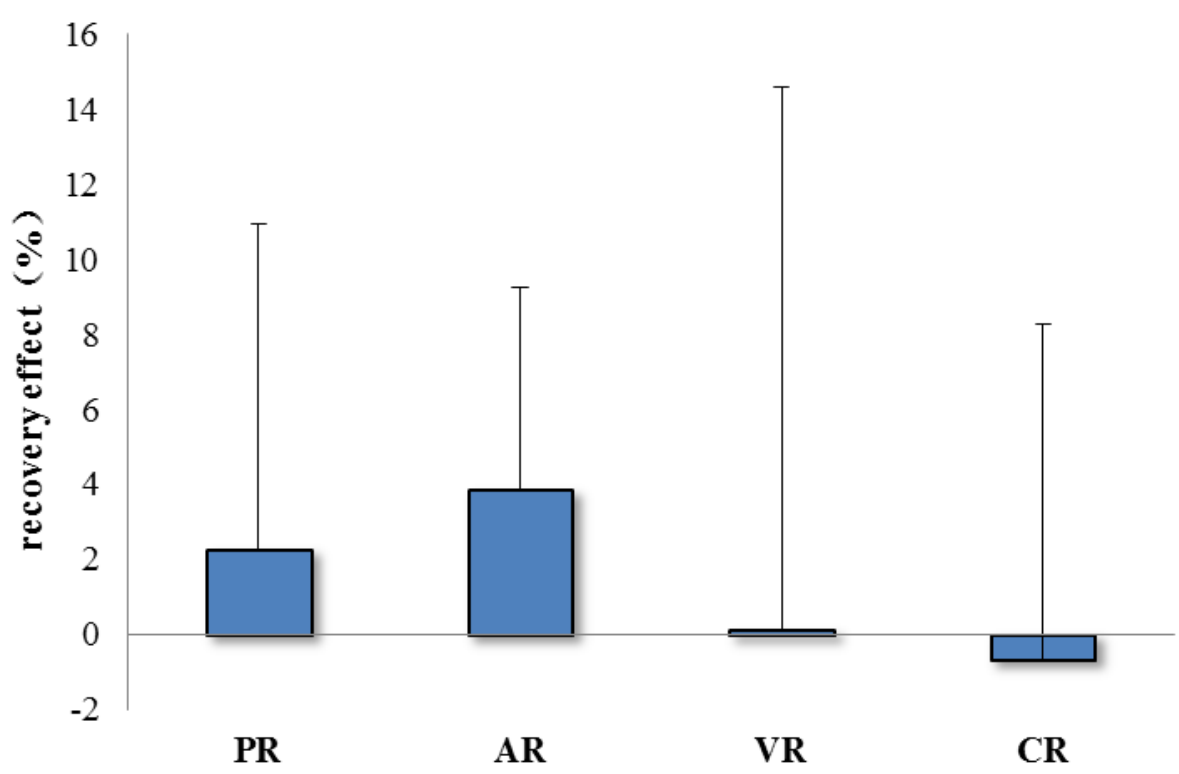

Fig.1 Comparison of the recovery effect of MS

\section{Summary}

It is indicated in the researches that AR has little effect on MS recovery after muscle fatigue, and there is no significant difference on effect of four recovery ways. MS refers to deformation degree of muscles at the time of being subject to external pressure, and it is found in this research that MS at different time pints varies little. Its possible reason is that fatigue exercise does not cause significant difference on MS in short time, or sensitivity degree of muscle tone meter used in this research is insufficient. This instrument were mostly used on special patients[4] in past researches, such as recovery situation and judgment on muscle stiffness of muscular paralysis and paralytic patients. Scholars[5] such as Murayama, etc ever researched the influence of muscle fatigue on MS, and they used weak hand BBM to execute maximal centrifugal contraction exercise for 24 times, then MS increased until the third day. It is assumed that centrifugal exercise does not lead to affection on MS immediately after causing muscle injury, so it may be difficult to distinguish the difference before and after recovery of muscle fatigue to use this instrument in this research.

Though difference of different recovery ways on MS influence is hard to be determined via experimental data, MS measurement data increases significantly after AR is found in this research, namely that AR has little effect on MS recovery. For muscle temperature is also a factor affecting MS, collagen flexibility of tendon and passively contraction tissues (such as myolemma and perimysium, etc) can also be increased after increasing muscle temperature [6]. Though muscle temperature is not directly measured in this research, continuous muscle contraction appears for AR, maybe causing high 
muscle temperature in four recovery ways. Thus malleability of soft tissues is increased, causing reduction of MS measurement value. While there is no obvious difference for CR before and after recovery, there is downtrend in terms of data of recovery effect, indicating MS increase may be linked with muscle temperature. So muscle temperature variation will be measured in future researches during recovery to offer more direct explanation on MS change.

\section{Acknowledgments}

This work was supported in part by the Project of the Fundamental Research Funds for the Central Universities under Grant LGZD201709, in part by the Project of China Postdoctoral Science Foundation under Grant 2017M611849, in part by Jiangsu Qing LAN Project under Grant 2017, and in part by Nanjing Forest police College Teaching Reform Project under Grant ZD17001 \& YB17001.

\section{References}

[1]. Ibrahim O, Omar H, Hamdi C, et al. Effects of Recovery Type after a Kickboxing Match on Blood Lactate and Performance in Anaerobic Tests. Asian Journal of Sports Medicine. Vol. 5 (2014) No. 2, p. 99-107.

[2]. Dupont G, Blondel N, Berthoin S. Performance for short intermittent runs: active recovery vs. passive recovery. European Journal of Applied Physiology. Vol. 89 (2003) No. 6, p.548-554.

[3]. ZHANG F, WANG C S, YE Z Q, et al. An Experimental Study Comparing the Effect of Different Stretching Mode on Hamstrings Flexibility. Journal of Tianjin University of Sport. Vol. 29 (2014) No. 1, p. 61-65. (In Chinese)

[4]. Arya K N, Pandian S. Effect of Task-Based Mirror Therapy on Motor Recovery of the Upper Extremity in Chronic Stroke Patients: A Pilot Study. Topics in Stroke Rehabilitation. Vol. 20 (2013) No. 3, p.210-217.

[5]. Murayama M, Nosaka K, Yoneda T, et al. Changes in hardness of the human elbow flexor muscles after eccentric exercise. European Journal of Applied Physiology. Vol. 82 (2000) No. 5, p.361-367.

[6]. WANG C Z, ZHENG Y P. Measurement of Muscle Stiffness Based on a Vibro-ultrasound Method. China Medical Devices. Vol. 26 (2011) No. 1, p.30-33. 